\title{
The contribution of specific class I and II histone deacetylases in the development of anxiety- related behavior in a mouse model of prenatal immune challenge
}

\author{
C.B.M. Jegers \\ Maastricht University \\ c.jegers@student.maastrichtuniversity.nl
}

\begin{abstract}
Several lines of evidence demonstrated that maternal infection during gestation might be an environmental risk factor for offspring to develop mood and anxiety disorders later in life. Previous experiments revealed that anxiety-related behavior, observed after prenatal immune challenge, is associated with increased histone deacetylase (HDAC) activity in adult female mice. Based on these findings, this study aims to investigate which class I and II HDAC isotypes might be involved in the development of the long-lasting consequences of prenatal immune challenge. Therefore, pregnant $\mathrm{C}_{57} \mathrm{BL} / 6 \mathrm{~J} \mathrm{RccH}$ sd inbred mice were intravenously injected with poly I:C (5 mg/kg bodyweight) at gestational day (GD) 17 to mimic a viral infection. Brains from adult offspring were homogenized and used for western blotting to analyse the relative protein levels of $\mathrm{HDAC}_{1}, 2,3,4,5$ and 6. Due to a lack of signal in $\mathrm{HDAC}_{1}, \mathrm{HDAC}_{3}$ and $\mathrm{HDAC}_{5}$, an irregular signal in $\mathrm{HDAC}_{2}$, and a weak signal in HDAC4 and HDAC6, the quality of the western blots was insufficient for protein quantification. Therefore, no subsequent comparison could be made between mice exposed to prenatal poly I:C and PBS in the current study.
\end{abstract}

\section{Keywords}

Prenatal immune challenge, poly I:C, epigenetics, histone deacetylases, HDACs, affective disorders, depression, anxiety disorders 


\section{Introduction}

Mood and anxiety disorders contribute to the most prevalent classes of disorders in the western population; in terms of lifetime prevalence, this corresponds to an estimate of $20.8 \%$ and $28.8 \%$ respectively (1). According to DSM-V, major depressive disorder is a heterogeneous disorder comprising a high variable set of symptoms, ranging from depressed mood to feelings of inappropriate guilt and recurrent thoughts of death $(2,3)$. Although various hypotheses have been proposed, a definite molecular mechanism of mood disorders remains unresolved. This lack of understanding impedes the development of effective therapeutic strategies, as underscored by the fact that less than half of the patients show complete remission after antidepressant treatment (4). To provide new therapeutic targets, this study aims to elucidate whether there is a neurodevelopmental origin for affective disorders, mediated by epigenetic modulation.

The monoamine hypothesis posits that depression originates from an imbalance in mainly serotonergic and noradrenergic neurotransmission (5). In line with this classic theory, depletion of the essential amino acid tryptophan (TRP) - the precursor of serotonin (5$\mathrm{HT}$ ) - may result in the development of depressive symptoms (6). As presented in Figure 1, pro-inflammatory cytokines can reduce 5 - $\mathrm{HT}$ levels by enhancing the enzyme indoleamine 2,3-dioxygenase (IDO), which is located in the lungs, placenta, blood and brain $(7,8)$. IDO converts TRP into kynurenine (KYN), which is able to pass the blood brain barrier when produced peripherally (9). Heyes et al. reported that microglia can metabolize KYN into the neurotoxic 3-hydroxykynurenine (3-OH-KYN), a pathway enhanced by immune activation, and quinolinic acid (QUIN) (10, 11).3-OH-KYN may induce neuronal cell death by generating reactive oxygen species, whereas additional neuronal damage is caused by QUIN, which is an excitotoxic $\mathrm{N}$-methyl-D-aspartate (NMDA) receptor agonist $(12,13)$. However, astrocytes are able to convert KYN into the NMDA receptor antagonist kynurenic acid (KA), which protects neurons against NMDA overstimulation induced by QUIN $(14,15)$. Accumulating evidence suggests that the balance between neurotoxic and neuroprotective metabolites is decisive in the development of affective disorders $(8,16)$. 


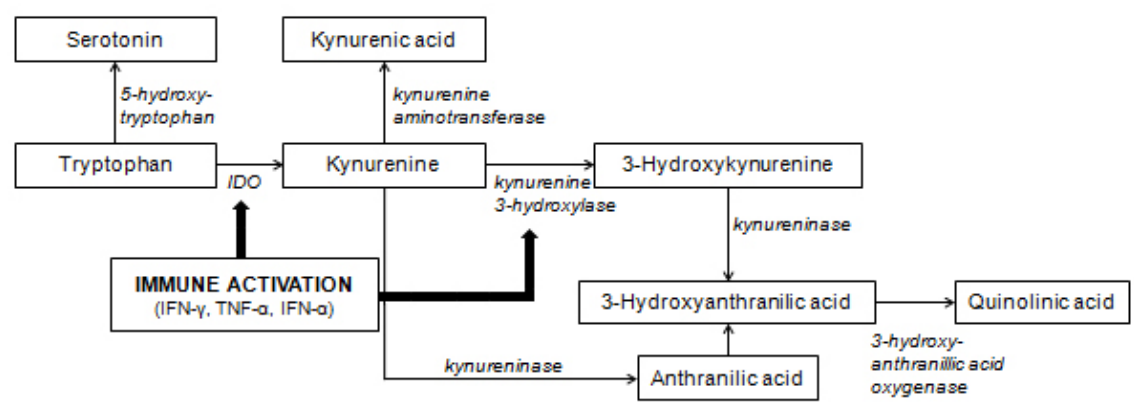

Figure 1. Kynurenine pathway. Immune activation promotes the conversion of tryptophan into kynurenine at the expense of serotonin synthesis. Kynurenine is either metabolized into the neuroprotective KA or into the neurodegenerative QUIN; the latter pathway is also stimulated by immune activation. IDO, indoleamine 2,3-dioxygenase. Adapted from Myint (11).

Recently, prenatal exposure to cytokines receives considerable interest, due to its ability to interfere with foetal brain development, which might result in long-lasting consequences. Foetal brain development is the result of complex dynamic events, ranging from neuronal birth to neuronal migration and synaptogenesis. Since these events depend on gene expression and environmental input, deviations in these factors may alter neuronal structure and function $(17,18)$. Canetta and Brown argue that activation of the maternal immune response may result in elevated cytokine levels in the foetus through direct (i.e. maternal sera) and/or indirect (i.e. activation of the foetal immune system) mechanisms $(19,20)$. In the foetal brain, these cytokines may interfere with neuronal development, as illustrated by interleukin-1 $\beta$ (IL-1 $\beta$ ), interleukin- 6 (IL-6) and tumour necrosis factor- $a$ (TNF- $\alpha$ ) which are able to affect neuronal survival and dendrite development $(21,22)$. Ultimately, this might result in the development of neuropathological and/or behavioral consequences later in life (19-22). In previous research, Pujol López and colleagues developed a mouse model of prenatal immune challenge in which mice were intravenously injected with polyriboinosinic-polyribocytidilic acid (poly I:C; synthetic analogue of viral doublestranded RNA) at gestational day (GD) 17, which corresponds to second-to-third trimester of human pregnancy. This group demonstrated that female offspring of immune challenged mice showed increased anxiety-related behavior in the open field test. Intriguingly, within this poly I:C stimulated group, female offspring expressed increased histone deacetylase (HDAC) activity - an enzyme involved in epigenetic gene silencing - compared to female controls (data unpublished).

Epigenetics refers to heritable changes in DNA or associated proteins, while preserving 
the underlying base-pair sequence (14). In addition to being essential in development and differentiation, epigenetic marks are present in mature mammals, either random or as a result of environmental exposure (23). For this reason, the study of epigenetics can be seen as the interface between genotype, phenotype and environment. The structural units of chromatin are nucleosomes, in which DNA is wrapped around a protein core consisting of two copies of four globular core histones. These core histones contain N-terminal amino acid tails which are prone to post-translational modification (e.g. histone acetylation), affecting the higher-order chromatin structure (24). HDACs catalyze histone deacetylation, leading to chromatin condensation. In promoter regions, chromatin condensation is often associated with gene inactivation (25). Members of the mammalian HDAC family are subdivided into four main classes, in which class I (HDAC1, 2,3 and 8) and II (HDAC4, 5, 6, 7, 9) are the most prominent in the nervous system (26).

As demonstrated by Pujol López et al., it is suggested that anxiety-related behavior observed after prenatal immune challenge, is mediated by epigenetic histone deacetylation. To investigate which HDAC isotypes might be involved in the development of the observed anxiety-related phenotype, the offspring's brains (obtained from the study conducted by Pujol López et al.) were homogenized and used for western blotting to analyse the relative protein levels of $\operatorname{HDAC}_{1}, 2,3,4,5$ and 6 . Based on current literature, it is hypothesized that HDAC2, 4, 5 and 6 might be involved in the development of affective disorders in the prenatal immune challenge mouse model.

\section{Material and methods}

\section{Animal model of prenatal immune challenge}

Male and female $\mathrm{C}_{57} \mathrm{BL} / 6 \mathrm{JRccHsd}$ inbred mice (Harlan Laboratories, Germany) were housed per four (single-sex) in conventional climate-controlled cages (55\% humidity, $22^{\circ} \mathrm{C}$; Tecniplast) according to EU guidelines. The cages were positively pressurized and ventilated. The mice were kept under a reversed 12-hour light-dark cycle with food (22\% protein, $4.5 \%$ fat; Sniff Spezialdiäten, Germany) and water ( $\mathrm{pH}$ 7.0) ad libitum. After three days of coupling, male and female mice were separated. In general, offspring was born after 21 days and separated from the mother three weeks after birth. All experimental procedures were approved by the government and the veterinarian administration of Oberbayern (AZ: 55.2-1-54-2531-61-10).

Pregnant mice were assigned to two groups; 20 pregnant mice received intravenous poly I:C injection ( $5 \mathrm{mg} / \mathrm{kg}$ bodyweight; Sigma-Aldrich, Germany), and 20 pregnant mice received intravenous phosphate buffered saline injection (PBS; $5 \mathrm{ml} / \mathrm{kg}$ bodyweight; $\mathrm{NaCl}$, 
$\mathrm{KCl}, \mathrm{Na}_{2} \mathrm{HPO}_{4}, \mathrm{KH}_{2} \mathrm{PO}_{4}, \mathrm{H}_{2} \mathrm{O}$; Sigma-Aldrich, Germany) as control. In this study, brains from 18 mice (9 male, 9 female) of the offspring from poly I:C stimulated mice, and 18 mice (gmale, 9 female) of the offspring from PBS treated mice, were used for HDAC immunoblotting.

Fourteen weeks after birth, male and female offspring was anesthetised by intraperitoneal injection containing $100 \mu \mathrm{l} / \mathrm{g}$ bodyweight narcotics ( $2 \%$ xylacin, $10 \%$ ketamin, $\mathrm{NaCl}$ ) and sacrificed by cervical dislocation. The brains were dissected and stored in RNAlater ${ }^{\circledR}$ (Qiagen, Germany).

\section{First western blot pilot}

Since the brains from the prenatal immune challenge mouse model were unavailable at the start of the pilot studies, fresh frozen brain tissue from an unrelated mouse model was used for the first western blot pilot. The brain tissue was mechanically homogenized using the Mini-Beadbeater ${ }^{\text {TM }}$ (Biospec, The Netherlands). Lysis buffer (PBS, 1\% igepal, 0.1\% triton, $1 \%$ glycerol, $1 \mathrm{mM}$ EDTA, 1mM EGTA, protease cocktail in Milli-O) was used to dissolve the cell membranes and inhibit proteolytic enzymes which could damage target HDACs. After determination of the protein concentration by Lowry protein assay (Bio-Rad, The Netherlands), samples were dissolved in sample buffer ( $1 \mathrm{M}$ Tris $\mathrm{HCl} \mathrm{pH} \mathrm{6.8,75 \%} \mathrm{glycerol,}$ SDS, $\beta$-mercaptoehtanol, brome phenol blue in Milli-O) and boiled for 7 minutes at $100^{\circ} \mathrm{C}$. A $10 \%$ SDS-PAGE gel was used for electrophoresis at $100 \mathrm{~V}$ and - after proteins passed the stacking gel - at $150 \mathrm{~V}$. The gel consisted of acrylamide/bis, tris $\mathrm{HCl}(3 \mathrm{M} \mathrm{pH} 8.8$ in running gel; $1 \mathrm{M} \mathrm{pH} 6.8$ in stacking gel) and $20 \%$ SDS. The polymerization of acrylamide/bis was initiated by $10 \%$ APS and catalyzed by TEMED. The transfer buffer contained glycine, trizma base and SDS dissolved in Milli-O. Next, a perpendicular oriented electric field (100 V, 90 minutes) was used to transfer the negatively charged proteins from the gel to a nitrocellulose membrane. The transfer buffer consisted of glycine and trizma base dissolved in Milli-O. Hydrophobic interactions facilitated the binding of the proteins to the nitrocellulose membrane (27). After three times of washing with PBS, blocking buffer (1:1 in PBS; Odyssey) blocked nonspecific binding sites and promoted renaturation of antigenic sites during one hour of incubation at room temperature on the shaker (28). The blots incubated overnight with primary antibodies specific to HDAC1, 2, 3, 4, 5, and 6 (1:1,000; \#9928, \#7612; Cell Signaling Technology) and GAPDH (1:2,000,000; Fitzgerald) on parafilm at $4^{\circ} \mathrm{C}$. After the membranes were washed once with PBS 0.1\% Tween and twice with PBS, the blots were incubated with secondary antibodies; goat anti rabbit (Alexa 800; 1:10,000; LI-COR) and donkey anti mouse (Alexa 680; 1:10,000; LI-COR) on the shaker at room temperature for one hour. The membranes were washed (PBS 0.1\% Tween, PBS, PBS) and 
protein bands were visualized using the Odyssey Scanner (LI-COR). Images were optimized using application software version 2.1 (Odyssey Infrared Imaging System).

\section{Second western blot pilot}

Since the protocol of the second pilot study is identical to the protocol used in the first pilot study, it will not be described separately. In contrast to the first pilot study, this experiment used two fresh frozen brains derived from female offspring of mice stimulated with either poly I:C or PBS.

In addition to the brains derived from the prenatal immune challenge mouse model, an extra sample was loaded into the SDS-PAGE gel to analyze the protein level of HDAC6. As this sample was used in the first western blot pilot, it served as a positive control in the second pilot study. Moreover, due to negative results obtained in the first western blot pilot, it was decided not to test the $\mathrm{HDAC}_{5}$ antibody in this experiment.

\section{Results}

\section{First western blot pilot}

A pilot study was performed to test and optimize a western blot protocol, which aimed to analyse the relative protein levels of $\operatorname{HDAC}_{1}, 23,4,5$ and 6 in brain tissue. In the first pilot study, three samples were used which contained brain stem and upper part of the spinal cord from an unrelated mouse model. As presented in Figure 2, all blots - including the negative control-expressed a nonspecific band at $25 \mathrm{kDa}$, caused by nonspecific binding of the secondary donkey anti-mouse antibody. Furthermore, a weak signal specific for HDAC1 and HDAC 3 was detected. This might be attributed to an ineffective transfer, masking of the antigen by blocking buffer, or simply because the samples contained a low HDAC1 and $\mathrm{HDAC}_{3}$ antigen concentration. In contrast to $\mathrm{HDAC}_{1}$ and $\mathrm{HDAC}_{3}$, a clear $\mathrm{HDAC}_{2}$ signal was observed. However, the flaw in this blot is that the expression of the housekeeping gene GAPDH is decreased compared to the other blots. This might be explained by an uneven agitation of primary antibodies as a consequence of their incubation on parafilm. In addition to their antigen specific bands, various nonspecific bands were detected in the blots specific to HDAC4 and HDAC6. The specificity of the monoclonal HDAC4 and HDAC6 antibodies is questioned, since the height of some these nonspecific bands correspond to HDAC expression observed in other blots. Proteolytic degradation and post-translational modification of $\mathrm{HDAC}_{4}$ and HDAC6 provide an additional explanation for the appearance of these unexpected bands. The protein bands in the HDAC4 blot are not flat, indicating that the proteins migrated too fast during electrophoresis. Thick bands, as observed in the 
HDAC6 blot, can be the result of ineffective stacking of the samples. Moreover, no signal was detected in the $\mathrm{HDAC}_{5}$ blot.

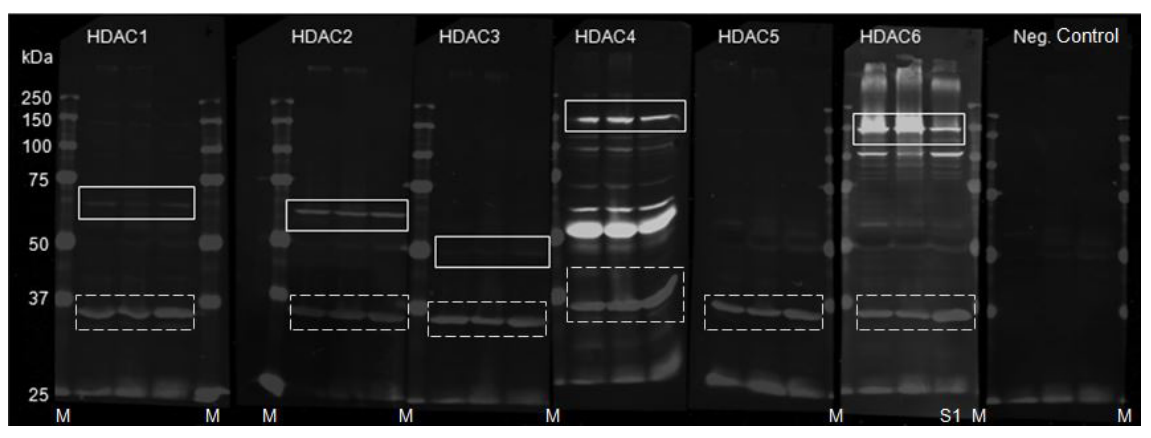

Figure 2. Results of the first western blot pilot using three different samples containing brain tissue from an unrelated mouse model. Based on the molecular weight $(\mathrm{kDa})$ of the Odyssey marker $(\mathrm{M})$, the relative expression of HDAC1, 2, 3, 4, 5, and 6 is indicated per three samples (solid lines). GAPDH expression is used as positive control (intermittent lines). Sample $\mathrm{S} 1$ is used as positive control in the second pilot study.

Moving forward, future pilot studies should test different primary antibody concentrations to optimize the contrast between specific HDAC signal and background signal - in particular in HDAC1, HDAC3, HDAC4 and HDAC6 blot. Although it is less cost-efficient, blots could be incubated with primary antibody in boxes without parafilm to prevent uneven agitation of the antibodies, as observed in the HDAC2 blot. Additionally, to prevent fast migration of the proteins in the $\mathrm{HDAC}_{4}$ blot, gel electrophoresis could be performed in the cold room $\left(4^{\circ} \mathrm{C}\right)$. Future directions specific to the HDAC5 blot involve testing of a HDAC 5 antibody from a different batch (obtained from Cell Signaling Technology, the manufacturer) and the use of a positive control, for example a purified $\mathrm{HDAC}_{5}$ antigen.

\section{Second western blot pilot}

A second western blot pilot was performed to analyse the protein levels of $\mathrm{HDAC}_{1}, 2,3,4$ and 6 in brains derived from offspring of mice exposed to poly I:C or PBS. As presented in Figure 3 , no protein bands specific to HDAC1 and HDAC3 were detected, which might be attributed to inadequate protein extraction by SDS. Furthermore, a weak specific signal was observed in the HDAC4 and HDAC6 blots. Since the signal of the marker was not observed at high molecular weights ( $>100 \mathrm{kDa}$ ), these findings might be the result of an ineffective transfer of proteins with high molecular weights. This theory is strengthened by the results obtained from the positive control (brain tissue from first pilot study), in 
which the signal specific to HDAC6 and GAPDH appeared to be reduced compared to the first pilot study (Figure 2, Figure 3; see S1). Although the western blot protocol was identical for both pilot studies, the protein transfer might have been less effective in the second pilot study. In future pilot studies, this experiment should be repeated, in which a positive control is used for each HDAC. In contrast to HDAC1, 3, 4, and 6, a clear HDAC2 signal was detected. However, the protein band observed in the poly I:C group is irregular, which might be caused by air bubbles trapped between the gel and the nitrocellulose membrane during transfer (29).

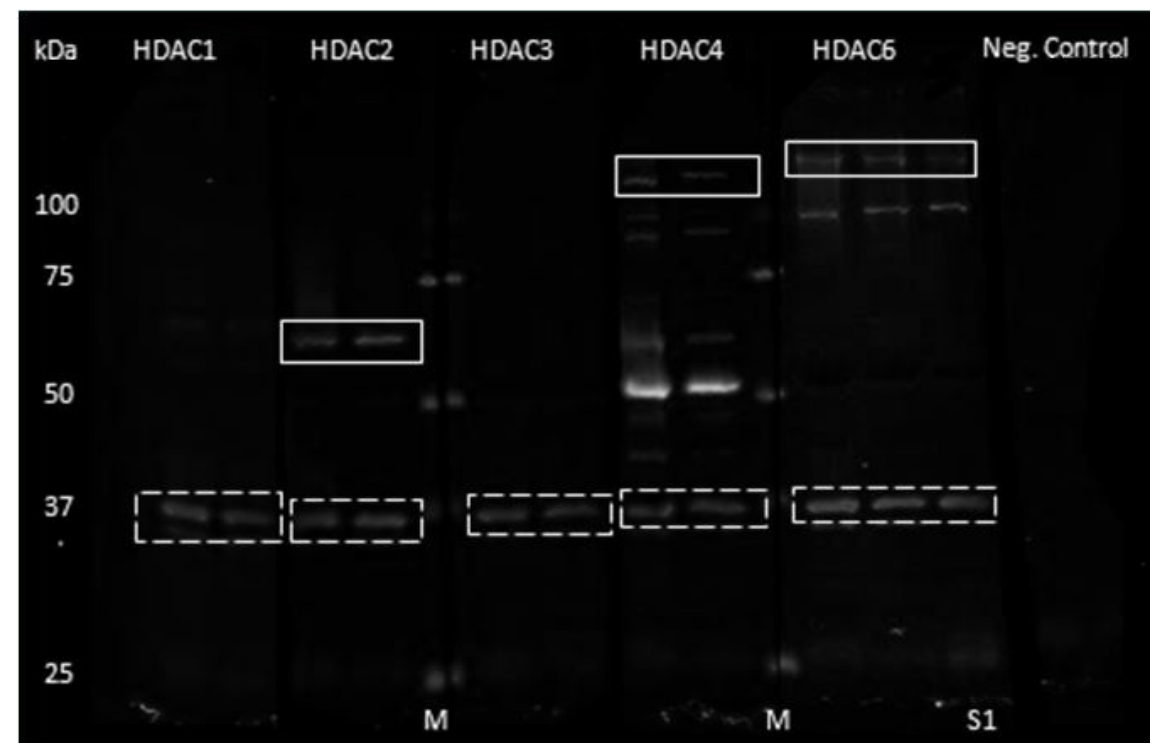

Figure 3. Results of the second western blot pilot using homogenized brains from offspring of mice exposed to prenatal poly I:C or PBS at GD17. Based on the molecular weight ( $\mathrm{kDa}$ ) of the Odyssey marker $(\mathrm{M})$, the relative expression of $\mathrm{HDAC1}, 2,3,4$ and 6 is indicated per two samples (left poly I:C, right PBS; solid lines); except in the HDAC6 blot, in which an additional sample from first pilot (S1) was used. GAPDH expression is used as positive control (intermittent lines).

\section{Discussion}

This study aimed to elucidate which class I and II HDAC isotypes might be involved in the development of anxiety-related behavior, observed after prenatal immune challenge. Therefore, homogenized brains of prenatal immune challenged mice were used for western blotting, to analyse the protein levels of HDAC1, 2, 3, 4, 5 and 6 . Due to a lack 
of signal in $\mathrm{HDAC}_{1}, \mathrm{HDAC}_{3}$ and $\mathrm{HDAC}_{5}$, an irregular signal in $\mathrm{HDAC}_{2}$, and a weak signal in HDAC4 and HDAC6, the quality of the western blots was insufficient for protein quantification. Therefore, no subsequent comparison could be made between mice exposed to prenatal poly I:C and PBS in the current study. Instead, this section provides a theoretical framework relating histone remodelling to affective disorders in the context of prenatal immune challenge.

After administration, poly I:C binds to toll-like receptor (TLR) 3 which stimulates the maternal viral-like acute phase response mediated by various cytokines, presumably IL-1 $\beta$, IL-6 and TNF-a, as presented in Figure 4 (30). Canetta and Brown argued that maternal immune activation may lead to elevated cytokine levels in the foetus (19). After crossing the blood brain barrier, the cytokines might exert detrimental effects on brain development through direct and indirect mechanisms, including the production of oxidative stress and activation of the kynurenine pathway. In addition to the (in)direct effects of cytokines, the maternal immune response can adversely affect foetal brain development via alternative mechanisms. Immune activation is often accompanied by hypothalamic prostaglandin $\mathrm{E}$ $\left(P G E_{2}\right)$ synthesis which might induce fever. Together with the induction of neuropeptidemediated anorexia, this might result in potent metabolic deficiencies in the foetus (31). Accumulating evidence suggests that prenatal malnutrition increases the risk to develop neuropsychiatric disorders later in life, reviewed by Markham and Koenig (18). Additionally, foetal oxygen supply is impaired during maternal infection as a result of restricted placental blood flow. Consequently, oxygen deprivation can further stimulate cytokine production or exert its detrimental effects via the production of reactive oxygen species (18). Furthermore, the maternal immune response plays an important role in stimulating the hypothalamic-pituitary-adrenal (HPA) axis, which results in elevated levels of glucocorticoids (GCs) in pregnant mice. After crossing the placenta, GCs can interfere with neuronal development, for example by inhibiting neurogenesis and synaptogenesis (32). Therefore, to attribute the neurobehavioral effects observed in prenatal immune challenged mice, to cytokines; the consequences of prenatal malnutrition, hypoxia and hypersecretion of GCs on foetal brain development should be considered. 


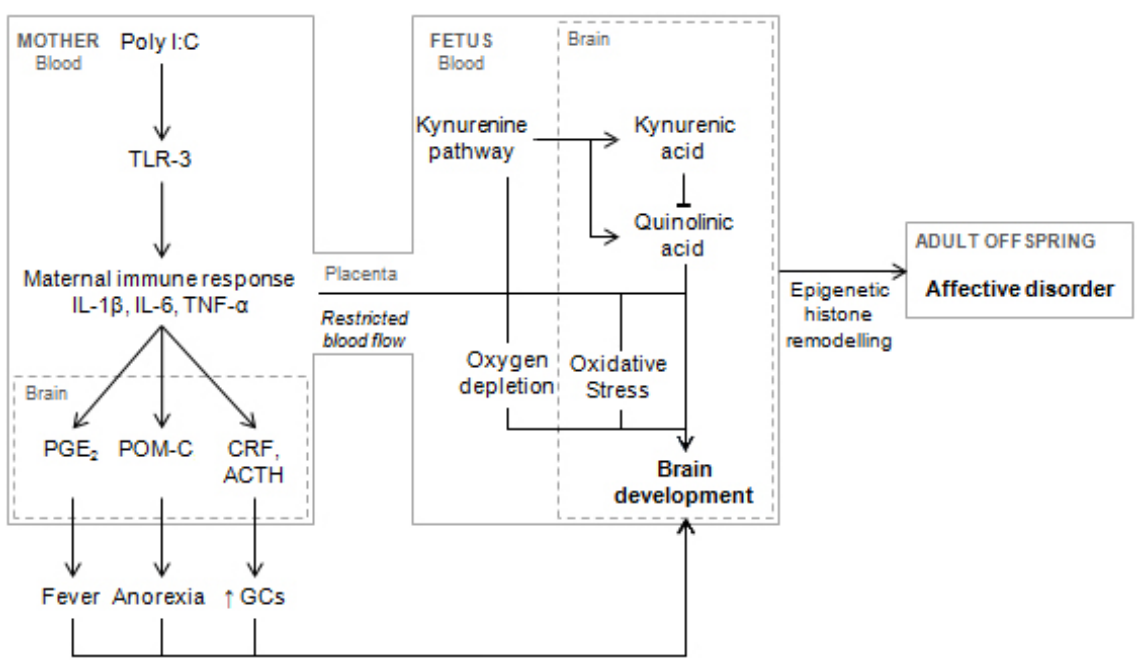

Figure 4. Proposed mechanism of the development of affective disorders in a mouse model of prenatal immune challenge. After administration, poly I:C binds to TLR-3, which activates the maternal immune response. Pro-inflammatory cytokines, including IL-1 $\beta$, IL- 6 and TNF-a, are generated and enter the foetal circulation. These cytokines might directly or indirectly (e.g. via the production of oxidative stress and activation of the kynurenine pathway) affect foetal brain development. In addition, immune activation is accompanied by $\mathrm{PGE}_{2}$-mediated fever and POM-C -mediated anorexia, both resulting in metabolic deficiencies in the foetus. Furthermore, the maternal immune response might impair placental blood flow - leading to foetal oxygen depletion - and it might stimulate the HPA-axis, leading to hypersecreted GCs. Together, prenatal malnutrition, hypoxia and GC hypersecretion affect foetal brain development. This study suggests that the subsequent development of affective disorders later life might be mediated by epigenetic histone remodelling. Abbreviations: ACTH, adrenocorticotrophic hormone; CRF, corticotrophin releasing factor; GCS, glucocorticoids; IL- intrleukin; poly I:C, polyriboinosinic-polyribocytidilic acid; PGE ${ }_{2}$, prostaglandin E; POM-C, pro-opiomelanocortin; TLR-3, toll-like receptor; TNF, tumour necrosis factor. Modified from Boksa (31).

Interest in histone remodelling as potential mediator in the development of mood and anxiety disorders, has grown considerably following the antidepressant-like effects observed after administration of various HDAC inhibitors (HDACi) (33). For example, Covington et al. demonstrated that anhedonia, observed in a chronic social defeat mouse model, is reversed after MS-275 (selective class I HDACi) infusion in the hippocampus, whereas social avoidance is reversed after MS-275 infusion in the amygdala (34). Still, the role of individual HDAC isotypes in the development of affective disorders remains to a large extent unclear. By affecting corticotrophin releasing hormone $(\mathrm{CRH})$ gene expression, Miller et al. suggested that HDAC1 might be involved in the negative feedback regulation of the HPA axis; a mechanism commonly disturbed in depression (35). However, no changes 
in depressive-like behavior were reported after hippocampal HDAC1 overexpression, in a study conducted by Bahari-Javan et al. (36). By down-regulating the glucocorticoid receptor (GR), Massart et al. developed a transgenic mouse model of depression (GR-i mouse model) in which the feedback control of the HPA axis is disrupted in the frontal cortex and the hippocampus (5). Massart's GR-i mutants expressed decreased mRNA levels of HDAC2 and $\mathrm{HDAC}_{5}$, which is thought to compensate for the loss of GR-i function. Tsankova et al. strengthened this hypothesis, by demonstrating that imipramine (tricyclic antidepressant) selectively down-regulates $\mathrm{HDAC}_{5}$ expression in a mouse model of chronic social defeat in the hippocampus (33). In line with Massart's findings, decreased protein levels of HDAC2 were observed in the nucleus accumbens (NAc) of both chronic social defeated mice and post-mortem brains of patients diagnosed with depression (37). Moreover, research by Kim et al. revealed that, compared to wild-type mice, mice with conditional HDAC4 deletion in brain, expressed decreased anxiety-related behavior in the elevated plus maze and the open field test; effects that were not observed in HDAC5 knockout mice (38). Furthermore, HDAC6-selective inhibitors seem to have antidepressant-like properties, which might involve acetylation of non-histone proteins, including a-tubulin (target of HDAC6) (39). In conclusion, to accurately attribute histone remodelling to the observed anxiety-related behavior, various caveats must be kept in mind when interpreting data obtained from the mouse model of prenatal immune challenge. Moreover, current literature suggests a functional role for $\operatorname{HDAC}_{1}, 2,4,5$ and 6 in the development of mood and anxiety disorders, which might provide promising strategies in treating affective disorders. However, it remains unclear whether these findings also apply to the development of anxiety-related behavior observed in the prenatal immune challenge mouse model. Taken together, further research is required to fully elucidate the contribution of epigenetic modulation in affective disorders in the mouse model of prenatal immune challenge.

\section{Role of the student}

C.B.M. Jegers was an undergraduate student in BioMedical Sciences working under the supervision of Yara Pujol López, Dr. Daniël van den Hove and Dr. Bart Rutten when the research in this report was performed. The topic was proposed by the supervisors. The student was involved in conducting the pilot studies and in processing of the results. Formulation of the conclusion and the writing were performed by the student.

\section{Acknowledgments}

I would like to thank Yara Pujol López, who guided me enthusiastically throughout my internship and supported me with her knowledge. I would like to thank Dr. Daniël van den 
Hove for his constructive suggestions during the development of this work. My grateful thanks are extended to Dr. Bart Rutten, Dr. Aye Mu Myint and Prof. Dr. Harry Steinbusch for sharing their valuable ideas. In addition, this work would not have been possible without the technical support of Marjan Philippens, Hellen Steinbusch and Denise Hermes. I would also like to thank Ehsan Pishva and Konstantinos Kompotis, who kindly provided brain tissue for our pilot studies.

\section{References}

1. Kessler RC, Berglund P, Demler O, Jin R, Merikangas KR, Walters EE. Lifetime prevalence and age-of-onset distributions of DSM-IV disorders in the National Comorbidity Survey Replication. Arch Gen Psychiatry. 2005;62(6):593.

2. Association AP. Diagnostic and Statistical Manual of Mental Disorders, Fifth Edition Arlington, VA: American Psychiatric Association; 2013. 155-88 p.

3. Gorman JM. Comorbid depression and anxiety spectrum disorders. Depress Anxiety. 1996;4(4):160-8

4. Berton O, Nestler EJ. New approaches to antidepressant drug discovery: beyond monoamines. Nat Rev Neurosci. 2006;7(2):137-51.

5. Massart R, Mongeau R, Lanfumey L. Beyond the monoaminergic hypothesis: neuroplasticity and epigenetic changes in a transgenic mouse model of depression. Phil Trans R Soc B. 2012;367(1601):2485-94

6. Young SN, Smith SE, Pihl RO, Ervin FR. Tryptophan depletion causes a rapid lowering of mood in normal males. Psychopharmacology. 1985;87(2):173-7.

7. Mellor AL, Munn DH. Tryptophan catabolism and T-cell tolerance: immunosuppression by starvation? Immunol Today. 1999;20(10):469-73.

8. Myint A-M, Kim YK, Verkerk R, Scharpé S, Steinbusch H, Leonard B. Kynurenine pathway in major depression: evidence of impaired neuroprotection. J Affect Disord. 2007;98(1):143-51.

9. Fukui S, Schwarcz R, Rapoport SI, Takada Y, Smith QQR. Blood-brain barrier transport of kynurenines: implications for brain synthesis and metabolism. J Neurochem. 1991;56(6):2007-17.

10. Heyes M, Achim C, Wiley C, Major E, Saito K, Markey S. Human microglia convert I-tryptophan into the neurotoxin quinolinic acid. Biochem J. 1996;320:595-7.

11. Myint AM. Kynurenines: from the perspective of major psychiatric disorders. FEBS J. 2012;279(8):1375-85.

12. Okuda S, Nishiyama N, Saito H, Katsuki H. 3-Hydroxykynurenine, an endogenous oxidative stress generator, causes neuronal cell death with apoptotic features and region selectivity. J Neurochem. 1998;70(1):299-307.

13. Wu H-Q, Guidetti P, Goodman J, Varasi M, Ceresoli-Borroni G, Speciale C, et al. Kynurenergic manipulations influence excitatory synaptic function and excitotoxic vulnerability in the rat hippocampus in vivo. Neuroscience. 2000;97(2):243-51.

14. Perkins $M$, Stone $T$. An iontophoretic investigation of the actions of convulsant kynurenines and their interaction with the endogenous excitant quinolinic acid. Brain Res. 1982;247(1):184-7.

15. Guillemin GJ, Kerr SJ, Smythe GA, Smith DG, Kapoor V, Armati PJ, et al. Kynurenine pathway metabolism in human astrocytes: a paradox for neuronal protection. J Neurochem. 2001;78(4):842-53.

16. Wichers MC, Koek G, Robaeys G, Verkerk R, Scharpé S, Maes M. IDO and interferon-a-induced depressive symptoms: a shift in hypothesis from tryptophan depletion to neurotoxicity. Mol Psychiatry. 2004;10(6):538-44

17. Stiles J, Jernigan TL. The basics of brain development. Neuropsychol Rev. 2010;20(4):327-48.

18. Markham JA, Koenig Jl. Prenatal stress: role in psychotic and depressive diseases. Psychopharmacology. 2011;214(1):89-106. 
19. Canetta SE, Brown AS. Prenatal infection, maternal immune activation, and risk for $\mathrm{s}$ chizophrenia. TransI Neurosci. 2012;3(4):320-7.

20. Howerton CL, Bale TL. Prenatal programing: at the intersection of maternal stress and immune activation. Horm Behav. 2012;62(3):237-42.

21. Gilmore JH, Jarskog LF, Vadlamudi S, Lauder JM. Prenatal infection and risk for schizophrenia: IL-1b, IL-6, and TNFa inhibit cortical neuron dendrite development. Neuropsychopharmacol. 2004;29:1221-9.

22. Jarskog LF, Xiao H, Wilkie MB, Lauder JM, Gilmore JH. Cytokine regulation of embryonic rat dopamine and serotonin neuronal survival in vitro. Int J Dev Neurosci. 1997;15(6):711-6.

23. Jaenisch R, Bird A. Epigenetic regulation of gene expression: how the genome integrates intrinsic and environmental signals. Nat Genet. 2003;33:245-54.

24. Kouzarides T. Chromatin modifications and their function. Cell. 2007;128(4):693-705.

25. Tsankova N, Renthal W, Kumar A, Nestler EJ. Epigenetic regulation in psychiatric disorders. Nat Rev Neurosci. 2007;8(5):355-67.

26. Abel T, Zukin RS. Epigenetic targets of HDAC inhibition in neurodegenerative and psychiatric disorders. Curr Opin Pharmacol. 2008;8(1):57-64.

27. MacPhee DJ. Methodological considerations for improving Western blot analysis. J Pharmacol Toxicol Methods. 2010;61(2):171-7.

28. Towbin H, Gordon J. Immunoblotting and dot immunobinding-current status and outlook. J Immunol Methods. 1984;72(2):313-40.

29. Mahmood T, Yang P-C. Western blot: Technique, theory, and trouble shooting. N Am J Med Sci. 2012;4(9):429.

30. Cunningham C, Campion S, Teeling J, Felton L, Perry V. The sickness behavior and CNS inflammatory mediator profile induced by systemic challenge of mice with synthetic double-stranded RNA (poly I: C). Brain Behav Immun. 2007;21(4):490-502.

31. Boksa P. Effects of prenatal infection on brain development and behavior: a review of findings from animal models. Brain Behav Immun. 2010;24(6):881-97.

32. Warner-Schmidt JL, Duman RS. Hippocampal neurogenesis: opposing effects of stress and antidepressant treatment. Hippocampus. 2006;16(3):239-49.

33. Tsankova NM, Berton O, Renthal W, Kumar A, Neve RL, Nestler EJ. Sustained hippocampal chromatin regulation in a mouse model of depression and antidepressant action. Nat Neurosci. 2006;9(4):519-25.

34. Covington III HE, Vialou VF, LaPlant Q, Ohnishi YN, Nestler EJ. Hippocampal-dependent antidepressant-like activity of histone deacetylase inhibition. Neurosci Lett. 2011;493(3):122-6.

35. Miller L, Foradori CD, Lalmansingh AS, Sharma D, Handa RJ, Uht RM. Histone deacetylase 1 (HDAC1) participates in the down-regulation of corticotropin releasing hormone gene (crh) expression. Physiol Behav. 2011;104(2):312-20.

36. Bahari-Javan S, Maddalena A, Kerimoglu C, Wittnam J, Held T, Bähr M, et al. HDAC1 regulates fear extinction in mice. J Neurosci. 2012;32(15):5062-73.

37. Covington HE, Maze I, LaPlant OC, Vialou VF, Ohnishi YN, Berton O, et al. Antidepressant actions of histone deacetylase inhibitors. J Neurosci. 2009;29(37):11451-60.

38. Kim M-S, Akhtar MW, Adachi M, Mahgoub M, Bassel-Duby R, Kavalali ET, et al. An essential role for histone deacetylase 4 in synaptic plasticity and memory formation. J Neurosci. 2012;32(32):10879-86.

39. Jochems J, Boulden J, Lee BG, Blendy JA, Jarpe M, Mazitschek R, et al. Antidepressant-Like Properties of Novel HDAC6-Selective Inhibitors with Improved Brain Bioavailability. Neuropsychopharmacology. 2013. 